Article

\title{
Asymptotic Convergence of the Solution of a Singularly Perturbed Integro-Differential Boundary Value Problem
}

\author{
Assiya Zhumanazarova and Young Im Cho * \\ Department of Computer Engineering, Gachon University, Gyeonggi-do 461-701, Korea; \\ assiya_mukhamaddin@mail.ru \\ * Correspondence: yicho@gachon.ac.kr; Tel.: +82-10-3267-4727
}

Received: 3 January 2020; Accepted: 6 February 2020; Published: 7 February 2020

check for updates

\begin{abstract}
In this study, the asymptotic behavior of the solutions to a boundary value problem for a third-order linear integro-differential equation with a small parameter at the two higher derivatives has been examined, under the condition that the roots of the additional characteristic equation are negative. Via the scheme of methods and algorithms pertaining to the qualitative study of singularly perturbed problems with initial jumps, a fundamental system of solutions, the Cauchy function, and the boundary functions of a homogeneous singularly perturbed differential equation are constructed. Analytical formulae for the solutions and asymptotic estimates of the singularly perturbed problem are obtained. Furthermore, a modified degenerate boundary value problem has been constructed, and it was stated that the solution of the original singularly perturbed boundary value problem tends to this modified problem's solution.
\end{abstract}

Keywords: small parameter; singular perturbation; boundary functions; asymptotic estimates

\section{Introduction}

Mathematical models of real processes provide important and necessary data, considering the features of the studied objects. To ensure the reliability of the data obtained via investigation, the model should be available for analytical and numerical processing. To simplify the differential equations used in the models, factors that exert insignificant influence on the process are often not taken into account. The actual roles of these small factors, and their effects on the process, determine the level of reliability of the data obtained from the associated model. To ensure the effectiveness of the results, an extended model is considered that includes the factors that had been omitted in the simplified model, and the solutions obtained via the two models are compared. The extended model utilizes differential equations with small parameters at the higher derivatives. To study this boundary value problem for integro-differential equations with a small parameter in the two higher derivatives, we need to briefly review the general theory of singularly perturbed problems and the methods that have been developed to solve these problems to determine the effectiveness of the chosen method for this task.

Differential equations with small parameters occur in mathematical models for multiple problems studied in various fields of natural science. Such problems can be classified into two groups-regularly perturbed problems and singularly perturbed ones, which differ in the impact of perturbations on their solutions. Unperturbed models utilize the original equations, which do not contain perturbations or terms with small parameters. Regular perturbation changes the solutions of the unperturbed problem slightly, and singular perturbation changes them significantly. The primary concern of perturbation theory is whether the difference between the solutions of the perturbed and unperturbed problems tends to zero when a small parameter tends to zero [1]. 
The most important aspects of perturbation theory were discussed and considered from a mathematical perspective in the works of Friedrichs [2] and Kato [3]. The investigation of equations with a small parameter in higher derivatives, called singularly perturbed equations, is important because of their range of applicability in models for various processes. In singularly perturbed problems, the dependence of solutions on small parameters is studied. The works of Tikhonov $[4,5]$ on the theory of differential equations with small parameters at the derivatives significantly influenced the effective progress of research in singular perturbation theory. In these papers, the fundamental theorems on limit transition for a system of nonlinear ordinary differential equations with a small parameter in the higher derivatives were proved.

In the theory of singular perturbations, effective methods exist to solve and investigate associated problems. However, in many practical problems, it is often impossible to apply classical methods to obtain their exact solutions. Thus, it is necessary to develop various methods for the approximate construction of solutions, with asymptotic methods being the primary ones. Asymptotic methods are used to find approximate solutions to singularly perturbed problems and to obtain formulae describing the asymptotic behavior of the solution when the small parameter tends to zero. An important asymptotic method is the construction of asymptotic expansions of solutions to singularly perturbed boundary value problems with respect to small values of the parameter. The underlying principle of this method lies in the reduction of the problem that is being considered to two simpler, already studied problems, by using solutions whose asymptotic representations yield the sought-after solution. Lomov [6-8] developed the regularization method for singularly perturbed problems by replacing the original singularly perturbed operator with a regular operator. The main idea of the regularization method is to move to a higher dimensional space by introducing additional independent variables.

For many singularly perturbed problems, rapid changes in the solution in specific regions such as boundary layers are characteristic. The method of boundary functions was developed by Vishik and Lyusternik [9] for singularly perturbed linear differential equations, by Vasilyeva [10] for singularly perturbed nonlinear ordinary differential equations, and by Imanaliev [11,12] for singularly perturbed integro-differential systems. The method of corner boundary functions developed by Butuzov $[13,14]$ constructs the asymptotic of solutions of differential equations using partial derivatives in domains whose boundaries contain corner points. The work of Vazov [15] was devoted to the development of methods of asymptotic expansion for ordinary differential equations and the study of singularly perturbed boundary value problems.

Research in the field of singularly perturbed problems has often been motivated by the practical occurrences of such problems. Examples include the introduction of the concept of the boundary layer in hydrodynamics and the development of computing devices, as well as the theory of automatic control and nonlinear oscillations, gas dynamics, quantum mechanics, and chemical kinetics. Mathematical formulations of several problems, for example problems related to the investigation of underground hydromechanics and hydrodynamic parameters of the formation, can be reduced to the singularly perturbed boundary value problems. Nayfe [16] considered methods using small parameters for application in problems of mechanics and mathematical physics, such as relaxation oscillations, flow around a body, and thermo-elastic surface waves. Cole [17] studied various methods in perturbation theory to solve associated physical and technical problems. The methods that were considered, including different-scale expansions, singular perturbation problems to partial differential equations, boundary layer theory in a viscous incompressible flow, problems of a magneto-hydrodynamic flow in a pipe, and a viscous boundary layer in a rotating fluid, represent the general approach of perturbation theories.

Asymptotic methods are used to solve certain problems encountered in physics and engineering. Asymptotic expansion in the degree of a small parameter is an effective tool for the study of nonlinear oscillations. In practical cases, it is an important method to obtain comparative calculation schemes and determine the characters of oscillatory processes. Bogolyubov and Mitropolsky [18] developed an averaging method to solve problems of nonlinear oscillations. Mishchenko and Rozov [19] developed 
a method to calculate relaxation oscillations described by using singularly perturbed nonlinear systems of ordinary differential equations.

In the theory of singular perturbations, problems with an initial jump are considered in order to study boundary value problems in cases where the fast variables of the initial sought-after function are not bounded when the small parameter tends to zero. In such problems, the solution to the unperturbed problem, when a small parameter tends to zero, tends to the solution of the perturbed equation with modified initial conditions. The phenomenon of the initial jump was investigated in the works of Vishik, Lyusternik [20], and Kasymov [21-23]. The works of Nurgabyl [24,25] were devoted to the study of boundary value problems for singularly perturbed differential equations. Dauylbaev [26-31] considered singularly perturbed initial and boundary value problems with initial jumps for linear and nonlinear integro-differential equations.

The importance of investigating asymptotic methods in singularly perturbed problems for integro-differential equations lies in the fact that they allow us to analyze the behavior of solutions and increase their accuracy when such equations are used in mathematical models to describe various processes. In this paper, we study the asymptotic behavior of the two-point boundary value problem for a linear integro-differential equation of the third order with a small parameter at two higher derivatives, when the roots of the additional characteristic equation are negative. The purpose of this work is to determine whether the difference between the solutions of the perturbed and unperturbed problem tends to zero when a small parameter tends to zero. Based on the above works, this paper makes the following contributions related to the solution of a singularly perturbed boundary value problem for a third order integro-differential equation, when the roots of the additional characteristic equation are negative: (1) Definition of an analytical formula and asymptotic estimates; (2) Identification of the phenomenon of the initial jump; (3) Detection of the significant influence of integral terms; and (4) Establishment of the asymptotic convergence of the solution of the original problem to the solution of the modified degenerate boundary value problem containing the initial jumps of both the solution and the integral.

The rest of the paper is organized as follows. A singularly perturbed integro-differential boundary value problem and asymptotic representations of the fundamental system of solutions, the Cauchy function, and the boundary functions are presented in Section 2. Section 3 is devoted to the derivation of asymptotic estimates of the solution of a singularly perturbed boundary value problem. Finally, Section 4 provides some conclusions.

\section{Problem Formulation and Auxiliary Results}

In this section, we present a problem formulation and some auxiliary results that are used in the rest of the paper.

\subsection{Problem Formulation}

Consider a singularly perturbed third-order linear integro-differential equation:

$$
\begin{aligned}
L_{\varepsilon} y & \equiv \varepsilon^{2} y^{\prime \prime \prime}+\varepsilon A(t) y^{\prime \prime}+B(t) y^{\prime}+C(t) y= \\
& =F(t)+\int_{0}^{1}\left[K_{0}(t, x) y(x, \varepsilon)+K_{1}(t, x) y^{\prime}(x, \varepsilon)\right] d x
\end{aligned}
$$

subject to boundary conditions:

$$
\begin{aligned}
& h_{1} y(t, \varepsilon) \equiv y(0, \varepsilon)=\alpha, \\
& h_{2} y(t, \varepsilon) \equiv y^{\prime}(0, \varepsilon)=\beta, \\
& h_{3} y(t, \varepsilon) \equiv y(1, \varepsilon)=\gamma,
\end{aligned}
$$

where $\varepsilon>0$ is a small parameter, and $\alpha, \beta, \gamma$ are known constants.

Suppose that the following conditions are true: 
1. The functions $A(t), B(t), C(t), F(t)$ in the segment $0 \leq t \leq 1$ and $K_{i}(t, x), i=0,1$ in the domain $D=\{0 \leq t \leq 1,0 \leq x \leq 1\}$ are infinitely differentiable.

2. The functions $\mu_{i}(t), i=1,2$ are distinct non-zero roots of the equation

$$
\mu^{2}+A(t) \mu+B(t)=0
$$

The roots of Equation (3) satisfy the conditions

$$
\mu_{i}(t) \leq-\delta \equiv \text { const }<0, i=1,2,0 \leq t \leq 1 .
$$

3. The parameter $\lambda=1$ for a sufficiently small $\varepsilon$ is not an eigenvalue of the kernel

$$
J(t, p, \varepsilon)=\frac{1}{\varepsilon^{2}} \int_{0}^{1}\left[K_{0}(t, x) K(x, p, \varepsilon)+K_{1}(t, x) K^{\prime}(x, p, \varepsilon)\right] d x .
$$

\subsection{The Fundamental System of Solutions}

Consider a homogeneous equation corresponding to Equation (1):

$$
L_{\varepsilon} y \equiv \varepsilon^{2} y^{\prime \prime \prime}+\varepsilon A(t) y^{\prime \prime}+B(t) y^{\prime}+C(t) y=0 .
$$

The characteristic equation corresponding to Equation (4) is defined as follows:

$$
\varepsilon^{2} \lambda^{3}+\varepsilon A(t) \lambda^{2}+B(t) \lambda+C(t)=0 .
$$

Multiplying both sides of Equation (5) by $\varepsilon$, we make the substitution $\mu=\varepsilon \lambda$ :

$$
\mu^{3}+A(t) \mu^{2}+B(t) \mu+\varepsilon C(t)=0 .
$$

The roots of Equation (6) can be sought as a series:

$$
\mu(t, \varepsilon)=\mu(t)+\varepsilon \mu_{1}(t)+\ldots
$$

Substituting the series (7) into Equation (6), we obtain the equality:

$$
\left(\mu(t)+\varepsilon \mu_{1}(t)+\ldots\right)^{3}+A(t)\left(\mu(t)+\varepsilon \mu_{1}(t)+\ldots\right)^{2}+B(t)\left(\mu(t)+\varepsilon \mu_{1}(t)+\ldots\right)+\varepsilon C(t)=0 .
$$

Equating the coefficients corresponding to the zero-th degree of $\varepsilon$, we obtain Equation $(3)[9,26]$. We denote the roots of this equation by $\mu_{1}(t), \mu_{2}(t)$, and these roots satisfy conditions 2 . Then, the fundamental system of solutions of the homogeneous Equation (4) can be sought in the following form:

$$
\begin{aligned}
& y_{i}(t, \varepsilon)=e^{\frac{1}{\varepsilon} \int_{0}^{t} \mu_{i}(t, x) d x}\left(y_{i 0}(t)+\varepsilon y_{i 0}(t)+\ldots\right), i=1,2, \\
& y_{3}(t, \varepsilon)=y_{30}(t)+\varepsilon y_{30}(t)+\ldots
\end{aligned}
$$

Substituting formula (8) into Equation (4) and equating the coefficients corresponding to the zero-th degree of $\varepsilon$, we obtain the following problems for the coefficients $y_{i 0}(t), y_{30}(t), i=1,2$ :

$$
\begin{aligned}
& \left(2 \mu_{i}^{2}(t)+A(t) \mu_{i}(t)\right) y_{i 0}^{\prime}(t)+\left(3 \mu_{i}(t) \mu_{i}^{\prime}(t)+A(t) \mu_{i}^{\prime}(t)+C(t)\right) y_{i 0}(t)=0, \\
& y_{i 0}(0)=1, i=1,2
\end{aligned}
$$


and

$$
\begin{aligned}
& B(t) y_{30}^{\prime}(t)+C(t) y_{30}(t)=0, \\
& y_{30}(0)=1 .
\end{aligned}
$$

According to condition 3, by the theorems of Schlesinger, Birkhoff, and Noailon [6,32], the following asymptotic representations are valid for the fundamental system of solutions of the homogeneous Equation (4):

$$
\begin{aligned}
& y_{i}^{(j)}(t, \varepsilon)=\frac{1}{\varepsilon^{j}} e^{\frac{1}{\varepsilon} \int^{t} \mu_{i}(t, x) d x}\left(\mu_{i}^{j}(t) y_{i 0}(t)+\mathrm{O}(\varepsilon)\right), \\
& y_{3}^{(j)}(t, \varepsilon)=y_{30}(t)+\mathrm{O}(\varepsilon), i=1,2, j=\overline{0,2},
\end{aligned}
$$

where $\mu_{i}(t), i=1,2$ are the roots of the Equation (3), and the coefficients $y_{i 0}(t), i=1,2,3$, are the solutions of the problems (9) and (10), respectively.

\subsection{Cauchy Function}

We introduce the Cauchy function $K(t, p, \varepsilon), 0 \leq p \leq t \leq 1$, as a solution of the following problem $[24,26]$ :

$$
\begin{aligned}
& L_{\varepsilon} K(t, p, \varepsilon)=0, \\
& K(p, p, \varepsilon)=0, K^{\prime}(p, p, \varepsilon)=0, K^{\prime \prime}(p, p, \varepsilon)=1 .
\end{aligned}
$$

Then, the following theorem is valid.

Theorem 1. The Cauchy function $K(t, p, \varepsilon), 0 \leq p \leq t \leq 1$, exists, is unique, and is determined by the formula [23]:

$$
K(t, p, \varepsilon)=\frac{1}{W(p, \varepsilon)} W_{3}(t, p, \varepsilon),
$$

where $W(p, \varepsilon) \neq 0$ is Wronskian of the fundamental system of solutions $y_{i}(p, \varepsilon), i=1,2,3$, and $W_{3}(t, p, \varepsilon)$ is a determinant obtained from $W(p, \varepsilon)$ by replacing its third row with the fundamental system of solutions $y_{i}(t, \varepsilon), i=1,2,3$.

Proof. The function $K(t, p, \varepsilon)$ can be considered as a linear combination of the fundamental system of solutions $y_{i}(t, \varepsilon), i=1,2,3$ :

$$
K(t, p, \varepsilon)=C_{1}(p) y_{1}(t, \varepsilon)+C_{2}(p) y_{2}(t, \varepsilon)+C_{3}(p) y_{3}(t, \varepsilon) .
$$

Obviously, the linear combination (14) is the solution of the homogeneous Equation (4). Therefore, it is necessary to find the unknown constants $C_{i}(p), i=1,2,3$. As $y_{i}(t, \varepsilon), i=1,2,3$, comprise the fundamental system of solutions of a homogeneous equation, the Cauchy function, expressed as their linear combination, is also a solution to a homogeneous equation. To find them, applying the conditions from (12) to the equality (14), we obtain a system of linear algebraic equations:

$$
\begin{aligned}
K(p, p, \varepsilon) & =C_{1}(p) y_{1}(p, \varepsilon)+C_{2}(p) y_{2}(p, \varepsilon)+C_{3}(p) y_{3}(p, \varepsilon)=0 \\
K^{\prime}(p, p, \varepsilon) & =C_{1}(p) y_{1}^{\prime}(p, \varepsilon)+C_{2}(p) y_{2}^{\prime}(p, \varepsilon)+C_{3}(p) y_{3}^{\prime}(p, \varepsilon)=0 \\
K^{\prime \prime}(p, p, \varepsilon) & =C_{1}(p) y_{1}^{\prime \prime}(p, \varepsilon)+C_{2}(p) y_{2}^{\prime \prime}(p, \varepsilon)+C_{3}(p) y_{3}^{\prime \prime}(p, \varepsilon)=1
\end{aligned}
$$

Solving this system of equations using the Kramer method, we find constants $C_{i}(p), i=1,2,3$ :

$$
C_{1}(p)=\frac{1}{W(p, \varepsilon)}\left|\begin{array}{ll}
y_{2}(p, \varepsilon) & y_{3}(p, \varepsilon) \\
y_{2}^{\prime}(p, \varepsilon) & y_{3}^{\prime}(p, \varepsilon)
\end{array}\right|
$$




$$
\begin{gathered}
C_{2}(p)=-\frac{1}{W(p, \varepsilon)}\left|\begin{array}{ll}
y_{1}(p, \varepsilon) & y_{3}(p, \varepsilon) \\
y_{1}^{\prime}(p, \varepsilon) & y_{3}^{\prime}(p, \varepsilon)
\end{array}\right|, \\
C_{3}(p)=\frac{1}{W(p, \varepsilon)}\left|\begin{array}{ll}
y_{1}(p, \varepsilon) & y_{2}(p, \varepsilon) \\
y_{1}^{\prime}(p, \varepsilon) & y_{2}^{\prime}(p, \varepsilon)
\end{array}\right| .
\end{gathered}
$$

Substituting constants $C_{i}(p), i=1,2,3$ into equality (14), we obtain:

$$
\begin{aligned}
& K(t, p, \varepsilon)=\frac{1}{W(p, \varepsilon)} \quad\left\lceil\left|\begin{array}{ll}
y_{2}(p, \varepsilon) & y_{3}(p, \varepsilon) \\
y_{2}^{\prime}(p, \varepsilon) & y_{3}^{\prime}(p, \varepsilon)
\end{array}\right| \cdot y_{1}(t, \varepsilon)-\right. \\
& -\left|\begin{array}{ll}
y_{1}(p, \varepsilon) & y_{3}(p, \varepsilon) \\
y_{1}^{\prime}(p, \varepsilon) & y_{3}^{\prime}(p, \varepsilon)
\end{array}\right| \cdot y_{2}(t, \varepsilon)+ \\
& \left.+\left|\begin{array}{ll}
y_{1}(p, \varepsilon) & y_{2}(p, \varepsilon) \\
y_{1}^{\prime}(p, \varepsilon) & y_{2}^{\prime}(p, \varepsilon)
\end{array}\right| \cdot y_{3}(t, \varepsilon)\right]= \\
& =\frac{1}{W(p, \varepsilon)}\left|\begin{array}{lll}
y_{1}(p, \varepsilon) & y_{2}(p, \varepsilon) & y_{3}(p, \varepsilon) \\
y_{1}^{\prime}(p, \varepsilon) & y_{2}^{\prime}(p, \varepsilon) & y_{3}^{\prime}(p, \varepsilon) \\
y_{1}(t, \varepsilon) & y_{2}(t, \varepsilon) & y_{3}(t, \varepsilon)
\end{array}\right|=\frac{1}{W(p, \varepsilon)} W_{3}(t, p, \varepsilon) .
\end{aligned}
$$

Thus, it is proved that the Cauchy function is determined by formula (13). We can verify that the function expressed by formula (13) satisfies all the conditions of the Cauchy function.

Consider the following determinants:

$$
\begin{gathered}
W(p, \varepsilon)=\left|\begin{array}{lll}
y_{1}(p, \varepsilon) & y_{2}(p, \varepsilon) & y_{3}(p, \varepsilon) \\
y_{1}^{\prime}(p, \varepsilon) & y_{2}^{\prime}(p, \varepsilon) & y_{3}^{\prime}(p, \varepsilon) \\
y_{1}^{\prime \prime}(p, \varepsilon) & y_{2}^{\prime \prime}(p, \varepsilon) & y_{3}^{\prime \prime}(p, \varepsilon)
\end{array}\right|, \\
W_{3}^{(j)}(t, p, \varepsilon)=\left|\begin{array}{ccc}
y_{1}(p, \varepsilon) & y_{2}(p, \varepsilon) & y_{3}(p, \varepsilon) \\
y_{1}^{\prime}(p, \varepsilon) & y_{2}^{\prime}(p, \varepsilon) & y_{3}^{\prime}(p, \varepsilon) \\
y_{1}^{(j)}(t, \varepsilon) & y_{2}^{(j)}(t, \varepsilon) & y_{3}^{(j)}(t, \varepsilon)
\end{array}\right|, j=0,1,2 .
\end{gathered}
$$

By virtue of (11), Wronskian $W(p, \varepsilon)$ admits the following asymptotic approximation as $\varepsilon \rightarrow 0$ :

$$
W(p, \varepsilon)=\frac{1}{\varepsilon^{3}} e^{\frac{1}{\varepsilon} \int_{0}^{p}\left(\mu_{1}(x)+\mu_{2}(x)\right) d x}\left[y_{10}(p) y_{20}(p) y_{30}(p) \mu_{1}(p) \mu_{2}(p)\left(\mu_{2}(p)-\mu_{1}(p)\right)+\mathrm{O}(\varepsilon)\right] .
$$


For the determinants $W_{3}^{(j)}(t, p, \varepsilon), j=0,1,2$, the following asymptotic representations hold as $\varepsilon \rightarrow 0$ :

$$
\begin{aligned}
& W_{3}(t, p, \varepsilon)=\frac{1}{\varepsilon} e^{\frac{1}{\varepsilon} \int_{0}^{p}\left(\mu_{1}(x)+\mu_{2}(x)\right) d x}\left[-e^{\frac{1}{\varepsilon} \int_{p}^{t} \mu_{1}(x) d x} y_{10}(t) y_{20}(p) y_{30}(p) \mu_{2}(p)+\right. \\
& \left.+e^{\frac{1}{\varepsilon} \int_{p}^{t} \mu_{2}(x) d x} y_{10}(p) y_{20}(t) y_{30}(p) \mu_{1}(p)+y_{10}(p) y_{20}(p) y_{30}(t)\left(\mu_{2}(p)-\mu_{1}(p)\right)+\mathrm{O}(\varepsilon)\right] \text {, } \\
& W_{3}^{\prime}(t, p, \varepsilon)=\frac{1}{\varepsilon^{2}} e^{\frac{1}{\varepsilon} \int_{0}^{p}\left(\mu_{1}(x)+\mu_{2}(x)\right) d x}\left[-e^{\frac{1}{\varepsilon} \int_{p}^{t} \mu_{1}(x) d x} y_{10}(t) y_{20}(p) y_{30}(p) \mu_{1}(t) \mu_{2}(p)+\right. \\
& \left.+e^{\frac{1}{\varepsilon} \int_{p}^{t} \mu_{2}(x) d x} y_{10}(p) y_{20}(t) y_{30}(p) \mu_{2}(t) \mu_{1}(p)+\varepsilon y_{10}(p) y_{20}(p) y_{30}^{\prime}(t)\left(\mu_{2}(p)-\mu_{1}(p)\right)+\mathrm{O}\left(\varepsilon^{2}\right)\right] \text {, } \\
& W_{3}^{\prime \prime}(t, p, \varepsilon)=\frac{1}{\varepsilon^{3}} e^{\frac{1}{\varepsilon} \int_{0}^{p}\left(\mu_{1}(x)+\mu_{2}(x)\right) d x}\left[-e^{\frac{1}{\varepsilon} \int_{p}^{t} \mu_{1}(x) d x} y_{10}(t) y_{20}(p) y_{30}(p) \mu_{1}^{2}(t) \mu_{2}(p)+\right. \\
& +e^{\frac{1}{\varepsilon} \int_{p}^{t} \mu_{2}(x) d x} y_{10}(p) y_{20}(t) y_{30}(p) \mu_{2}^{2}(t) \mu_{1}(p)+ \\
& \left.+\varepsilon^{2} y_{10}(p) y_{20}(p) y_{30}^{\prime \prime}(t)\left(\mu_{2}(p)-\mu_{1}(p)\right)+\mathrm{O}\left(\varepsilon^{3}+\varepsilon e^{\frac{1}{\varepsilon} \int_{p}^{t} \mu_{1}(x) d x}+\varepsilon e^{\frac{1}{\varepsilon} \int_{p}^{t} \mu_{2}(x) d x}\right)\right] \text {. }
\end{aligned}
$$

Hence, according to Theorem 1 and representations (15) and (16), we obtain asymptotic representations for the Cauchy function:

$$
\begin{aligned}
& K(t, p, \varepsilon)=\varepsilon^{2}\left(\frac{y_{30}(t)}{y_{30}(p) \mu_{1}(p) \mu_{2}(p)}-\frac{y_{10}(t)}{y_{10}(p) \mu_{1}(p)\left(\mu_{2}(p)-\mu_{1}(p)\right)} e^{\frac{1}{\varepsilon} \int_{p}^{t} \mu_{1}(x) d x}+\right. \\
& \left.+\frac{y_{20}(t)}{y_{20}(p) \mu_{2}(p)\left(\mu_{2}(p)-\mu_{1}(p)\right)} e^{\frac{1}{\varepsilon} \int_{p}^{t} \mu_{2}(x) d x}\right)+\mathrm{O}(\varepsilon) \\
& K^{\prime}(t, p, \varepsilon)=\varepsilon^{2} \frac{y_{30}^{\prime}(t)}{y_{30}(p) \mu_{1}(p) \mu_{2}(p)}-\varepsilon \frac{y_{10}(t) \mu_{1}(t)}{y_{10}(p) \mu_{1}(p)\left(\mu_{2}(p)-\mu_{1}(p)\right)} e^{\frac{1}{\varepsilon} \int_{p}^{t} \mu_{1}(x) d x}+ \\
& +\varepsilon \frac{y_{20}(t) \mu_{2}(t)}{y_{20}(p) \mu_{2}(p)\left(\mu_{2}(p)-\mu_{1}(p)\right)} e^{\frac{1}{\varepsilon} \int_{p}^{t} \mu_{2}(x) d x}+\mathrm{O}\left(\varepsilon^{3}+\varepsilon^{2} e^{\frac{1}{\varepsilon} \int_{p}^{t} \mu_{1}(x) d x}+\varepsilon^{2} e^{\frac{1}{\varepsilon} \int_{p}^{t} \mu_{2}(x) d x}\right), \\
& K^{\prime \prime}(t, p, \varepsilon)=\varepsilon^{2} \frac{y_{30}^{\prime \prime}(t)}{y_{30}(p) \mu_{1}(p) \mu_{2}(p)}-\frac{y_{10}(t) \mu_{1}^{2}(t)}{y_{10}(p) \mu_{1}(p)\left(\mu_{2}(p)-\mu_{1}(p)\right)} e^{\frac{1}{\varepsilon} \int_{p}^{t} \mu_{1}(x) d x}+ \\
& +\frac{y_{20}(t) \mu_{2}^{2}(t)}{y_{20}(p) \mu_{2}(p)\left(\mu_{2}(p)-\mu_{1}(p)\right)} e^{\frac{1}{\varepsilon} \int_{p}^{t} \mu_{2}(x) d x}+\mathrm{O}\left(\varepsilon^{3}+\varepsilon e^{\frac{1}{\varepsilon} \int_{p}^{t} \mu_{1}(x) d x}+\varepsilon e^{\frac{1}{\varepsilon} \int_{p}^{t} \mu_{2}(x) d x}\right) .
\end{aligned}
$$

which admit the following asymptotic estimates:

$$
\left|K^{(j)}(t, p, \varepsilon)\right| \leq C \varepsilon^{2}+\frac{C}{\varepsilon^{j-2}} e^{-\delta \frac{t-p}{\varepsilon}}, j=0,1,2 .
$$

where $C>0, \delta>0$ are constants independent of $\varepsilon$. 


\subsection{Boundary Functions}

The boundary functions $\Phi_{i}(t, \varepsilon), i=1,2,3$, are defined to be the solutions of the problem:

$$
\begin{aligned}
& L_{\varepsilon} \Phi_{i}(t, \varepsilon)=0, \\
& h_{l} \Phi_{i}(t, \varepsilon)=\delta_{l i}, l, i=1,2,3 .
\end{aligned}
$$

where $\delta_{l i}$ is the Kronecker delta $[24,26]$.

Consider the determinant:

$$
\Delta(\varepsilon)=\left|\begin{array}{lll}
h_{1} y_{1}(t, \varepsilon) & h_{1} y_{2}(t, \varepsilon) & h_{1} y_{3}(t, \varepsilon) \\
h_{2} y_{1}(t, \varepsilon) & h_{2} y_{2}(t, \varepsilon) & h_{2} y_{3}(t, \varepsilon) \\
h_{3} y_{1}(t, \varepsilon) & h_{3} y_{2}(t, \varepsilon) & h_{3} y_{3}(t, \varepsilon)
\end{array}\right|
$$

By the fundamental system of solutions (11), the determinant $\Delta(\varepsilon)$ has the following asymptotic representation as $\varepsilon \rightarrow 0$

$$
\Delta(\varepsilon)=\frac{1}{\varepsilon}\left[\left(\mu_{2}(0)-\mu_{1}(0)\right) y_{30}(1)+\mathrm{O}(\varepsilon)\right]
$$

Then, the following theorem is valid:

Theorem 2. The boundary functions $\Phi_{i}(t, \varepsilon), i=1,2,3$, exist in the segment $0 \leq t \leq 1$, are unique, and are determined by the formula [23]:

$$
\Phi_{i}(t, \varepsilon)=\frac{1}{\Delta(\varepsilon)} \Delta_{i}(t, \varepsilon),
$$

where $\Delta_{i}(t, \varepsilon), i=\overline{1,3}$ are the determinants obtained from $\Delta(\varepsilon)$ by replacing its $i$-th row with the fundamental system of the solutions, $y_{i}(t, \varepsilon), i=1,2,3$.

Proof. We will search the boundary functions $\Phi_{i}(t, \varepsilon), i=1,2,3$, in the form:

$$
\Phi_{i}(t, \varepsilon)=c_{i 1}(\varepsilon) y_{1}(t, \varepsilon)+c_{i 2}(\varepsilon) y_{2}(t, \varepsilon)+c_{i 3}(\varepsilon) y_{3}(t, \varepsilon) .
$$

where $c_{i j}(\varepsilon), i, j=1,2,3$, are constants that depend only on $\varepsilon$. As equality (22) satisfies the linear homogeneous Equation (4), we merely need to find constants $c_{i j}(\varepsilon), i, j=1,2,3$, substituting $\Phi_{i}(t, \varepsilon), i=$ $1,2,3$, from formula (22) into conditions from (19). Consider the proof in the case of $i=1$ for the obtained system of linear algebraic equations:

$$
\begin{aligned}
& h_{1} \Phi_{1}(t, \varepsilon) \equiv c_{11} h_{1} y_{1}(t, \varepsilon)+c_{12} h_{1} y_{2}(t, \varepsilon)+c_{13} h_{1} y_{3}(t, \varepsilon)=1, \\
& h_{2} \Phi_{1}(t, \varepsilon) \equiv c_{11} h_{2} y_{1}(t, \varepsilon)+c_{12} h_{2} y_{2}(t, \varepsilon)+c_{13} h_{2} y_{3}(t, \varepsilon)=0, \\
& h_{3} \Phi_{1}(t, \varepsilon) \equiv c_{11} h_{3} y_{1}(t, \varepsilon)+c_{12} h_{3} y_{2}(t, \varepsilon)+c_{13} h_{3} y_{3}(t, \varepsilon)=0 .
\end{aligned}
$$


Applying the Kramer formula, we get:

$$
\begin{aligned}
& c_{11}=\frac{1}{\Delta(\varepsilon)}\left|\begin{array}{lll}
1 & h_{1} y_{2}(t, \varepsilon) & h_{1} y_{3}(t, \varepsilon) \\
0 & h_{2} y_{2}(t, \varepsilon) & h_{2} y_{3}(t, \varepsilon) \\
0 & h_{3} y_{2}(t, \varepsilon) & h_{3} y_{3}(t, \varepsilon)
\end{array}\right|=\frac{1}{\Delta(\varepsilon)}\left|\begin{array}{ll}
h_{2} y_{2}(t, \varepsilon) & h_{2} y_{3}(t, \varepsilon) \\
h_{3} y_{2}(t, \varepsilon) & h_{3} y_{3}(t, \varepsilon)
\end{array}\right|= \\
& =\frac{1}{\Delta(\varepsilon)}\left(h_{2} y_{2}(t, \varepsilon) h_{3} y_{3}(t, \varepsilon)-h_{3} y_{2}(t, \varepsilon) h_{2} y_{3}(t, \varepsilon)\right) \text {, } \\
& c_{12}=\frac{1}{\Delta(\varepsilon)}\left|\begin{array}{lll}
h_{1} y_{1}(t, \varepsilon) & 1 & h_{1} y_{3}(t, \varepsilon) \\
h_{2} y_{1}(t, \varepsilon) & 0 & h_{2} y_{3}(t, \varepsilon) \\
h_{3} y_{1}(t, \varepsilon) & 0 & h_{3} y_{3}(t, \varepsilon)
\end{array}\right|=-\frac{1}{\Delta(\varepsilon)}\left|\begin{array}{ll}
h_{2} y_{1}(t, \varepsilon) & h_{2} y_{3}(t, \varepsilon) \\
h_{3} y_{1}(t, \varepsilon) & h_{3} y_{3}(t, \varepsilon)
\end{array}\right|= \\
& =\frac{1}{\Delta(\varepsilon)}\left(h_{3} y_{1}(t, \varepsilon) h_{2} y_{3}(t, \varepsilon)-h_{2} y_{1}(t, \varepsilon) h_{3} y_{3}(t, \varepsilon)\right) \text {, } \\
& c_{13}=\frac{1}{\Delta(\varepsilon)}\left|\begin{array}{lll}
h_{1} y_{1}(t, \varepsilon) & h_{1} y_{2}(t, \varepsilon) & 1 \\
h_{2} y_{1}(t, \varepsilon) & h_{2} y_{2}(t, \varepsilon) & 0 \\
h_{3} y_{1}(t, \varepsilon) & h_{3} y_{2}(t, \varepsilon) & 0
\end{array}\right|=\frac{1}{\Delta(\varepsilon)}\left|\begin{array}{ll}
h_{2} y_{1}(t, \varepsilon) & h_{2} y_{2}(t, \varepsilon) \\
h_{3} y_{1}(t, \varepsilon) & h_{3} y_{2}(t, \varepsilon)
\end{array}\right|= \\
& =\frac{1}{\Delta(\varepsilon)}\left(h_{2} y_{1}(t, \varepsilon) h_{3} y_{2}(t, \varepsilon)-h_{3} y_{1}(t, \varepsilon) h_{2} y_{2}(t, \varepsilon)\right) \text {. }
\end{aligned}
$$

We substitute the formulae (24) into equality (22) for $i=1$ and obtain:

$$
\begin{aligned}
\Phi_{1}(t, \varepsilon) & =\frac{1}{\Delta(\varepsilon)}\left|\begin{array}{ll}
h_{2} y_{2}(t, \varepsilon) & h_{2} y_{3}(t, \varepsilon) \\
h_{3} y_{2}(t, \varepsilon) & h_{3} y_{3}(t, \varepsilon)
\end{array}\right| \cdot y_{1}(t, \varepsilon)- \\
& -\frac{1}{\Delta(\varepsilon)}\left|\begin{array}{cc}
h_{2} y_{1}(t, \varepsilon) & h_{2} y_{3}(t, \varepsilon) \\
h_{3} y_{1}(t, \varepsilon) & h_{3} y_{3}(t, \varepsilon)
\end{array}\right| \cdot y_{2}(t, \varepsilon)+ \\
& +\frac{1}{\Delta(\varepsilon)}\left|\begin{array}{ccc}
h_{2} y_{1}(t, \varepsilon) & h_{2} y_{2}(t, \varepsilon) \\
h_{3} y_{1}(t, \varepsilon) & h_{3} y_{2}(t, \varepsilon)
\end{array}\right| \cdot y_{3}(t, \varepsilon)= \\
& =\frac{1}{\Delta(\varepsilon)}\left|\begin{array}{ccc}
y_{1}(t, \varepsilon) & y_{2}(t, \varepsilon) & y_{3}(t, \varepsilon) \\
h_{2} y_{1}(t, \varepsilon) & h_{2} y_{2}(t, \varepsilon) & h_{2} y_{3}(t, \varepsilon) \\
h_{3} y_{1}(t, \varepsilon) & h_{3} y_{2}(t, \varepsilon) & h_{3} y_{3}(t, \varepsilon)
\end{array}\right|=\frac{1}{\Delta(\varepsilon)} \Delta_{1}(t, \varepsilon) .
\end{aligned}
$$

Similarly, the proof can be carried out for the cases $i=2,3$. It can be verified that the functions defined by formula (21) satisfy all the conditions of the boundary functions for the boundary value problem (1), (2). Thus, Theorem 2 is proved.

Consider the following determinants:

$$
\begin{aligned}
\Delta_{1}^{(j)}(t, \varepsilon) & =\left|\begin{array}{ccc}
y_{1}^{(j)}(t, \varepsilon) & y_{2}^{(j)}(t, \varepsilon) & y_{3}^{(j)}(t, \varepsilon) \\
h_{2} y_{1}(t, \varepsilon) & h_{2} y_{2}(t, \varepsilon) & h_{2} y_{3}(t, \varepsilon) \\
h_{3} y_{1}(t, \varepsilon) & h_{3} y_{2}(t, \varepsilon) & h_{3} y_{3}(t, \varepsilon)
\end{array}\right|, \\
\Delta_{2}^{(j)}(t, \varepsilon) & =\left|\begin{array}{ccc}
h_{1} y_{1}(t, \varepsilon) & h_{1} y_{2}(t, \varepsilon) & h_{1} y_{3}(t, \varepsilon) \\
y_{1}^{(j)}(t, \varepsilon) & y_{2}^{(j)}(t, \varepsilon) & y_{3}^{(j)}(t, \varepsilon) \\
h_{3} y_{1}(t, \varepsilon) & h_{3} y_{2}(t, \varepsilon) & h_{3} y_{3}(t, \varepsilon)
\end{array}\right|, \\
\Delta_{3}^{(j)}(t, \varepsilon) & =\left|\begin{array}{ccc}
h_{1} y_{1}(t, \varepsilon) & h_{1} y_{2}(t, \varepsilon) & h_{1} y_{3}(t, \varepsilon) \\
h_{2} y_{1}(t, \varepsilon) & h_{2} y_{2}(t, \varepsilon) & h_{2} y_{3}(t, \varepsilon) \\
y_{1}^{(j)}(t, \varepsilon) & y_{2}^{(j)}(t, \varepsilon) & y_{3}^{(j)}(t, \varepsilon)
\end{array}\right|, j=\overline{0,2} .
\end{aligned}
$$


By virtue of the fundamental system of solutions (11), these determinants have the following asymptotic representations as $\varepsilon \rightarrow 0$ :

$$
\begin{aligned}
\Delta_{1}^{(j)}(t, \varepsilon) & =\frac{1}{\varepsilon^{j+1}} e^{\frac{1}{\varepsilon} \int_{0}^{t} \mu_{1}(x) d x}\left(y_{10}(t) y_{30}(1) \mu_{1}^{j}(t) \mu_{2}(0)+\mathrm{O}(\varepsilon)\right)- \\
& -\frac{1}{\varepsilon^{j+1}} e^{\frac{1}{\varepsilon} \int_{0}^{t} \mu_{2}(x) d x}\left(y_{20}(t) y_{30}(1) \mu_{2}^{j}(t) \mu_{1}(0)+\mathrm{O}(\varepsilon)\right) \\
\Delta_{2}^{(j)}(t, \varepsilon) & =-\frac{1}{\varepsilon^{j}} e^{\frac{1}{\varepsilon} \int_{0}^{t} \mu_{1}(x) d x}\left(y_{10}(t) y_{30}(1) \mu_{1}^{j}(t)+\mathrm{O}(\varepsilon)\right)+ \\
& +\frac{1}{\varepsilon^{j}} e^{\frac{1}{\varepsilon} \int^{t} \mu_{2}(x) d x}\left(y_{20}(t) y_{30}(1) \mu_{2}^{j}(t)+\mathrm{O}(\varepsilon)\right) \\
\Delta_{3}^{(j)}(t, \varepsilon) & =-\frac{1}{\varepsilon^{j+1}} e^{\frac{1}{\varepsilon} \int_{0}^{t} \mu_{1}(x) d x}\left(y_{10}(t) \mu_{1}^{j}(t) \mu_{2}(0)+\mathrm{O}(\varepsilon)\right)+ \\
& +\frac{1}{\varepsilon^{j+1}} e^{\frac{1}{\varepsilon} \int_{0}^{t} \mu_{2}(x) d x}\left(y_{20}(t) \mu_{2}^{j}(t) \mu_{1}(0)+\mathrm{O}(\varepsilon)\right)+ \\
& +\frac{1}{\varepsilon}\left(y_{30}^{(j)}(t)\left(\mu_{2}(0)-\mu_{1}(0)\right)+\mathrm{O}(\varepsilon)\right), j=\overline{0,2} .
\end{aligned}
$$

Hence, according to Theorem 2, in view of the asymptotic representations (20) and (26), the boundary functions $\Phi_{i}(t, \varepsilon), i=1,2,3$, can be expressed in the form:

$$
\begin{aligned}
\Phi_{1}^{(j)}(t, \varepsilon) & =\frac{y_{10}(t) \mu_{1}^{j}(t) \mu_{2}(0)}{\varepsilon^{j}\left(\mu_{2}(0)-\mu_{1}(0)\right)} e^{\frac{1}{\varepsilon} \int_{0}^{t} \mu_{1}(x) d x}- \\
& -\frac{y_{20}(t) \mu_{2}^{j}(t) \mu_{1}(0)}{\varepsilon^{j}\left(\mu_{2}(0)-\mu_{1}(0)\right)} e^{\frac{1}{\varepsilon} \int_{0}^{t} \mu_{2}(x) d x}+\mathrm{O}\left(\frac{1}{\varepsilon^{j-1}} e^{\frac{1}{\varepsilon} \int^{t} \mu_{1}(x) d x}+\frac{1}{\varepsilon^{j-1}} e^{\frac{1}{\varepsilon} \int_{0}^{t} \mu_{2}(x) d x}\right), \\
\Phi_{2}^{(j)}(t, \varepsilon) & =-\frac{y_{10}(t) \mu_{1}^{j}(t)}{\varepsilon^{j-1}\left(\mu_{2}(0)-\mu_{1}(0)\right)} e^{\frac{1}{\varepsilon} \int^{t} \mu_{1}(x) d x}+ \\
& +\frac{y_{20}(t) \mu_{2}^{j}(t)}{\varepsilon^{j-1}\left(\mu_{2}(0)-\mu_{1}(0)\right)} e^{\frac{1}{\varepsilon} \int_{0}^{t} \mu_{2}(x) d x}+\mathrm{O}\left(\frac{1}{\varepsilon^{j-2}} e^{\frac{1}{\varepsilon} \int_{0}^{t} \mu_{1}(x) d x}+\frac{1}{\varepsilon^{j}-2} e^{\frac{1}{\varepsilon} \int_{0}^{t} \mu_{2}(x) d x}\right), \\
\Phi_{3}^{(j)}(t, \varepsilon)= & \frac{y_{30}^{(j)}(t)}{y_{30}(1)}-\frac{y_{10}(t) \mu_{1}^{j}(t) \mu_{2}(0)}{\varepsilon y_{30}(1)\left(\mu_{2}(0)-\mu_{1}(0)\right)} e^{\frac{1}{\varepsilon} \int_{0}^{t} \mu_{1}(x) d x}+ \\
& +\frac{y_{20}(t) \mu_{2}^{j}(t) \mu_{1}(0)}{\varepsilon^{j} y_{30}(1)\left(\mu_{2}(0)-\mu_{1}(0)\right)} e^{\frac{1}{\varepsilon} \int_{0}^{t} \mu_{2}(x) d x}+\mathrm{O}\left(\varepsilon+\frac{1}{\varepsilon^{j-1}} e^{\frac{1}{\varepsilon} \int_{0}^{t} \mu_{1}(x) d x}+\frac{1}{\varepsilon^{j-1}} e^{\frac{1}{\varepsilon} \int_{0}^{t} \mu_{2}(x) d x}, j=\overline{0,2 .} .\right.
\end{aligned}
$$

The boundary functions (27) admit the following asymptotic estimates as $\varepsilon \rightarrow 0$ :

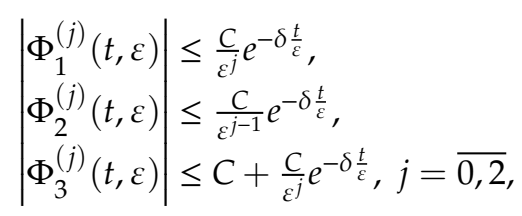

where $C>0, \delta>0$ are constants independent of $\varepsilon$.

\section{Solution of a Singularly Perturbed Boundary Value Problem}

In this section, to study the asymptotic behaviour of a solution, we consider asymptotic estimates of the solution and the difference of the solutions of singularly perturbed and unperturbed problems. 


\subsection{The Existence and Uniqueness Theorem of a Solution}

In the following theorem, we obtain an explicit analytical formula for the solution of the original boundary value problem (1), (2) using the Cauchy function and boundary functions constructed in Section 2. The original boundary value problem for integro-differential equations is reduced to the equivalent Fredholm integral equation. Since, by virtue of condition 3, this integral equation has a unique solution for small $\varepsilon$, then for these small $\varepsilon$ the original boundary value problem for integro-differential equations also has a unique solution. Constants $C_{i}, i=1,2,3$, are found from the boundary conditions (2).

Theorem 3. Suppose that conditions 1-3 are fulfilled. Then, for a sufficiently small $\varepsilon$, the solution of the boundary value problem (1), (2) exists in the segment [0,1], is unique, and can be expressed by the formula:

$$
\begin{aligned}
y(t, \varepsilon)= & \alpha Q_{1}(t, \varepsilon)+\beta Q_{2}(t, \varepsilon)+ \\
& +\frac{\gamma-\frac{1}{\varepsilon^{2}} \int_{0}^{1} K(1, p, \varepsilon)\left(\alpha \bar{\varphi}_{1}(p, \varepsilon)+\beta \bar{\varphi}_{2}(p, \varepsilon)+\bar{F}(p, \varepsilon)\right) d p}{1+\frac{1}{\varepsilon^{2}} \int_{0}^{1} K(1, p, \varepsilon) \bar{\varphi}_{3}(p, \varepsilon) d p} Q_{3}(t, \varepsilon)+P(t, \varepsilon),
\end{aligned}
$$

where

$$
\begin{aligned}
& Q_{i}(t, \varepsilon)=\Phi_{i}(t, \varepsilon)+\frac{1}{\varepsilon^{2}} \int_{0}^{t} K(t, p, \varepsilon) \bar{\varphi}_{i}(p, \varepsilon) d p \\
& P(t, \varepsilon)=\frac{1}{\varepsilon^{2}} \int_{0}^{t} K(t, p, \varepsilon) \bar{F}(p, \varepsilon) d p, \\
& \bar{\varphi}_{i}(p, \varepsilon)=\int_{0}^{1} \sum_{j=0}^{1} \bar{K}_{j}(p, x, \varepsilon) \Phi_{i}^{(j)}(x, \varepsilon) d x \\
& \bar{K}_{j}(p, x, \varepsilon)=K_{j}(p, x)+\int_{0}^{1} R(p, q, \varepsilon) K_{j}(q, x) d q \equiv \bar{K}_{j}(p, x)+\mathrm{O}(\varepsilon), \\
& \bar{F}(p, \varepsilon)=F(p)+\int_{0}^{1} R(p, q, \varepsilon) F(q) d q+\mathrm{O}(\varepsilon) \equiv \bar{F}(p)+\mathrm{O}(\varepsilon), i=\overline{1,3}, j=0,1,
\end{aligned}
$$

and $R(t, p, \varepsilon)$ is the resolvent of the kernel $J(t, p, \varepsilon)$.

Proof. We search the solution of the boundary value problem (1), (2) in the form:

$$
y(t, \varepsilon)=c_{1} \Phi_{1}(t, \varepsilon)+c_{2} \Phi_{2}(t, \varepsilon)+c_{3} \Phi_{3}(t, \varepsilon)+\frac{1}{\varepsilon^{2}} \int_{0}^{t} K(t, p, \varepsilon) F(p, \varepsilon) d p
$$

where $\Phi_{i}(t, \varepsilon), i=1,2,3$, are the boundary functions, $K(t, p, \varepsilon), 0 \leq p \leq t \leq 1$, is the Cauchy function, $c_{i}, i=1,2,3$, are the unknown constants, and $F(t, \varepsilon)$ is an unknown function to be defined. Substituting equality (31) into Equation (1) with respect to function $F(t, \varepsilon)$, we obtain the Fredholm integral equation of the second kind:

$$
F(t, \varepsilon)=f(t, \varepsilon)+\int_{0}^{t} J(t, p, \varepsilon) F(p, \varepsilon) d p,
$$


where:

$$
\begin{array}{r}
f(t, \varepsilon)=F(t)+c_{1} \int_{0}^{1}\left[K_{0}(t, x) \Phi_{1}(x, \varepsilon)+K_{1}(t, x) \Phi_{1}^{\prime}(x, \varepsilon)\right] d x+ \\
+c_{2} \int_{0}^{1}\left[K_{0}(t, x) \Phi_{2}(x, \varepsilon)+K_{1}(t, x) \Phi_{2}^{\prime}(x, \varepsilon)\right] d x+ \\
+c_{3} \int_{0}^{1}\left[K_{0}(t, x) \Phi_{3}(x, \varepsilon)+K_{1}(t, x) \Phi_{3}^{\prime}(x, \varepsilon)\right] d x \\
J(t, p, \varepsilon)=\frac{1}{\varepsilon^{2}} \int_{0}^{1}\left[K_{0}(t, x) K(x, p, \varepsilon)+K_{1}(t, x) K^{\prime}(x, p, \varepsilon)\right] d x .
\end{array}
$$

By virtue of condition 3, the integral equation (32) possesses a unique solution, which can be represented in the form:

$$
F(t, \varepsilon)=f(t, \varepsilon)+\int_{0}^{1} R(t, p, \varepsilon) f(p, \varepsilon) d p,
$$

where $R(t, p, \varepsilon)$ is the resolvent of the kernel $J(t, p, \varepsilon)$. Substituting formula (34) into equality (31) and considering the notations (33), we obtain the solution of the original problem in the form:

$$
y(t, \varepsilon)=\sum_{i=1}^{3} C_{i} Q_{i}(t, \varepsilon)+P(t, \varepsilon),
$$

where $Q_{i}(t, \varepsilon), P(t, \varepsilon)$ have the form (30). To determine $C_{i}, i=1,2,3$, we substitute formula (35) into boundary conditions (2) and obtain a system of algebraic equations:

$$
\begin{aligned}
& C_{1} Q_{1}(0, \varepsilon)+C_{2} Q_{2}(0, \varepsilon)+C_{3} Q_{3}(0, \varepsilon)=\alpha-P(0, \varepsilon), \\
& C_{1} Q_{1}^{\prime}(0, \varepsilon)+C_{2} Q_{2}^{\prime}(0, \varepsilon)+C_{3} Q_{3}^{\prime}(0, \varepsilon)=\beta-P^{\prime}(0, \varepsilon), \\
& C_{1} Q_{1}(1, \varepsilon)+C_{2} Q_{2}(1, \varepsilon)+C_{3} Q_{3}(1, \varepsilon)=\gamma-P(1, \varepsilon) .
\end{aligned}
$$

Hence, considering:

$$
\begin{aligned}
& \Phi_{1}(0, \varepsilon)=1, \Phi_{1}^{\prime}(0, \varepsilon)=0, \Phi_{1}(1, \varepsilon)=0, \\
& \Phi_{2}(0, \varepsilon)=0, \Phi_{2}^{\prime}(0, \varepsilon)=1, \Phi_{2}(1, \varepsilon)=0, \\
& \Phi_{3}(0, \varepsilon)=0, \Phi_{3}^{\prime}(0, \varepsilon)=0, \Phi_{3}(1, \varepsilon)=1,
\end{aligned}
$$

we obtain:

$$
\begin{aligned}
& C_{1}=\alpha, \\
& C_{2}=\beta, \\
& C_{3}=\frac{\gamma-\frac{1}{\varepsilon^{2}} \int_{0}^{1} K(1, p, \varepsilon)\left(\alpha \bar{\varphi}_{1}(p, \varepsilon)+\beta \bar{\varphi}_{2}(p, \varepsilon)+\bar{F}(p, \varepsilon)\right) d p}{1+\frac{1}{\varepsilon^{2}} \int_{0}^{1} K(1, p, \varepsilon) \bar{\varphi}_{3}(p, \varepsilon) d p} .
\end{aligned}
$$

Substituting the values (38) into formula (35), we obtain the final formula (29) for the solution of the problem (1), (2). Thus, Theorem 3 is proved.

By formula (30), in view of the asymptotic representations (17) and (27), we have the following asymptotic representations for $\bar{\varphi}_{i}(p, \varepsilon), i=1,2,3$, as $\varepsilon \rightarrow 0$ :

$$
\begin{aligned}
& \bar{\varphi}_{1}(p, \varepsilon)=-\bar{K}_{1}(p, 0)+\mathrm{O}(\varepsilon), \\
& \bar{\varphi}_{2}(p, \varepsilon)=-\varepsilon \frac{2 \bar{K}_{1}(p, 0)}{\mu_{2}(0)-\mu_{1}(0)}+\mathrm{O}\left(\varepsilon^{2}\right),
\end{aligned}
$$




$$
\bar{\varphi}_{2}(p, \varepsilon)=\int_{0}^{1} \sum_{j=0}^{1} \bar{K}_{j}(p, x) \frac{y_{30}^{(j)}(x)}{y_{30}(1)} d x+\frac{\bar{K}_{1}(p, 0)}{y_{30}(1)}+\mathrm{O}\left(\varepsilon+e^{\frac{1}{\varepsilon} \int_{0}^{t} \mu_{1}(x) d x}+e^{\frac{1}{\varepsilon} \int_{0}^{t} \mu_{2}(x) d x}\right) .
$$

Let the following condition be satisfied:

$$
\bar{\delta}=1+\int_{0}^{1} \frac{\bar{K}_{1}(p, 0)+\int_{0}^{1} \sum_{j=0}^{1} \bar{K}_{j}(p, x) y_{30}^{(j)}(x) d x}{y_{30}(p) \mu_{1}(p) \mu_{2}(p)} d p \neq 0 .
$$

Then, substituting the asymptotic representations (39) into equality (38) for $C_{3}$, we have an asymptotic representation in the form:

$$
C_{3}=\frac{\gamma-\int_{0}^{1} \frac{y_{30}(1)\left(\bar{F}(p)-\alpha \bar{K}_{1}(p, 0)\right)}{y_{30}(p) \mu_{1}(p) \mu_{2}(p)} d p}{1+\int_{0}^{1} \frac{\bar{K}_{1}(p, 0)+\int_{0}^{1} \sum_{j=0}^{1} \bar{K}_{j}(p, x) y_{30}^{(j)}(x) d x}{y_{30}(p) \mu_{1}(p) \mu_{2}(p)} d p}+\mathrm{O}(\varepsilon) .
$$

\subsection{Asymptotic Estimates of the Solution}

In order to study the asymptotic behavior of solutions of the singularly perturbed boundary value problem, it is necessary to determine the asymptotic estimates of the solutions and their derivatives.

So we have the following theorem:

Theorem 4. Suppose that conditions 1-4 are fulfilled. Then, the solution $y(t, \varepsilon)$ of the boundary value problem (1), (2) on the segment [0,1] has the following asymptotic estimates as $\varepsilon \rightarrow 0$ :

$$
\begin{aligned}
\left|y^{(j)}(t, \varepsilon)\right| & \leq C\left[|\gamma|+\varepsilon|\beta|+\max _{0 \leq t \leq 1}\left|F(t)-\alpha K_{1}(t, 0)\right|\right]+ \\
& +\frac{C}{\varepsilon} e^{-\delta \frac{t}{\varepsilon}}\left[\left(|\alpha|+|\gamma|+\max _{0 \leq t \leq 1}\left|F(t)-\alpha K_{1}(t, 0)\right|\right)\left|\mu_{2}(0) \mu_{1}^{j}(t)-\mu_{1}(0) \mu_{2}^{j}(t)\right|+\varepsilon|\beta|\right], j=\overline{0,2},
\end{aligned}
$$

where $C>0, \delta>0$ are constants independent of $\varepsilon$.

Proof. The following asymptotic representations hold true for the functions $Q_{i}(t, \varepsilon), P(t, \varepsilon)$ :

$$
\begin{aligned}
Q_{1}^{(j)}(t, \varepsilon) & =-\int_{0}^{t} \frac{\bar{K}_{1}(p, 0) y_{30}^{(j)}(t)}{y_{30}(p) \mu_{1}(p) \mu_{2}(p)} d p+\frac{y_{10}(t) \mu_{2}(0) \mu_{1}^{j}(t)}{\varepsilon j\left(\mu_{2}(0)-\mu_{1}(0)\right)} e^{\frac{1}{\varepsilon} \int_{0}^{t} \mu_{1}(x) d x}- \\
& -\frac{y_{20}(t) \mu_{1}(0) \mu_{2}^{j}(t)}{\varepsilon^{j}\left(\mu_{2}(0)-\mu_{1}(0)\right)} e^{\frac{1}{\varepsilon} \int_{0}^{t} \mu_{2}(x) d x}+\mathrm{O}\left(\varepsilon+\frac{1}{\varepsilon^{j-1}} e^{\frac{1}{\varepsilon} \int_{p}^{t} \mu_{1}(x) d x}+\frac{1}{\varepsilon^{j-1}} e^{\frac{1}{\varepsilon} \int_{p}^{t} \mu_{2}(x) d x}\right), \\
Q_{2}^{(j)}(t, \varepsilon)= & -\varepsilon \int_{0}^{t} \frac{2 \bar{K}_{1}(p, 0) y_{30}^{(j)}(t)}{y_{30}(p) \mu_{1}(p) \mu_{2}(p)\left(\mu_{2}(0)-\mu_{1}(0)\right)} d p+\frac{y_{10}(t) \mu_{1}^{j}(t)}{\varepsilon^{j-1}\left(\mu_{2}(0)-\mu_{1}(0)\right)} e^{\frac{1}{\varepsilon} \int_{0}^{t} \mu_{1}(x) d x}+ \\
& +\frac{y_{20}(t) \mu_{2}^{j}(t)}{\varepsilon^{j-1}\left(\mu_{2}(0)-\mu_{1}(0)\right)} e^{\frac{1}{\varepsilon} \int_{0}^{t} \mu_{2}(x) d x}+\mathrm{O}\left(\varepsilon+\frac{1}{\varepsilon^{j-2}} e^{\frac{1}{\varepsilon} \int_{p}^{t} \mu_{1}(x) d x}+\frac{1}{\varepsilon^{j-2}} e^{\frac{1}{\varepsilon} \int_{p}^{t} \mu_{2}(x) d x}\right),
\end{aligned}
$$




$$
\begin{aligned}
Q_{3}^{(j)}(t, \varepsilon)= & \frac{y_{30}^{(j)}(t)}{y_{30}(1)}+\int_{0}^{t} \frac{y_{30}^{(j)}(t)}{y_{30}(p) \mu_{1}(p) \mu_{2}(p) y_{30}(1)}\left(\bar{K}_{1}(p, 0)+\int_{0}^{1} \sum_{i=0}^{1} \bar{K}_{i}(p, x) y_{30}^{(i)}(x) d x\right) d p+ \\
& +\frac{y_{20}(t) \mu_{1}(0) \mu_{2}^{j}(t)}{\varepsilon^{j} y_{30}(1)\left(\mu_{2}(0)-\mu_{1}(0)\right)} e^{\frac{1}{\varepsilon} \int_{0}^{t} \mu_{2}(x) d x}-\frac{y_{10}(t) \mu_{2}(0) \mu_{1}^{j}(t)}{\varepsilon^{j} y_{30}(1)\left(\mu_{2}(0)-\mu_{1}(0)\right)} e^{\frac{1}{\varepsilon} \int_{0}^{t} \mu_{1}(x) d x}+ \\
+ & \mathrm{O}\left(\varepsilon+\frac{1}{\varepsilon^{j-1}} e^{\frac{1}{\varepsilon} \int^{p} \mu_{1}(x) d x}+\frac{1}{\varepsilon^{j-1}} e^{\frac{1}{\varepsilon} \int^{p} \mu_{2}(x) d x}\right) \\
P^{(j)}(t, \varepsilon)= & \int_{0}^{t} \frac{y_{30}^{(j)}(t) \bar{F}(p)}{y_{30}(p) \mu_{1}(p) \mu_{2}(p)} d p+\frac{\bar{F}(t)}{\varepsilon^{j-1}\left(\mu_{2}(t)-\mu_{1}(t)\right)}\left(\mu_{1}^{j-2}(t)-\mu_{2}^{j-2}(t)\right)- \\
& -\frac{y_{10}(t) \mu_{1}^{j}(t) \bar{F}(0)}{\varepsilon^{j-1} \mu_{1}^{2}(0)\left(\mu_{2}(0)-\mu_{1}(0)\right)} e^{\frac{1}{\varepsilon} \int_{0}^{t} \mu_{1}(x) d x}+\frac{y_{20}(t) \mu_{2}^{j}(t) \bar{F}(0)}{\varepsilon^{j-1} \mu_{2}^{2}(0)\left(\mu_{2}(0)-\mu_{1}(0)\right)} e^{\frac{1}{\varepsilon} \int_{0}^{t} \mu_{2}(x) d x}+ \\
& +\mathrm{O}\left(\varepsilon+\frac{1}{\varepsilon^{j-2}} e^{\frac{1}{\varepsilon} \int^{t} \mu_{1}(x) d x}+\frac{1}{\varepsilon^{j-2}} e^{\frac{1}{\varepsilon} \int_{p}^{p} \mu_{2}(x) d x} .\right.
\end{aligned}
$$

From formula (29), in view of equality (38) and the asymptotic representations (41), (43), and (44), we obtain the following asymptotic representations for the solution $y(t, \varepsilon)$ of the problem (1), (2):

$$
\begin{aligned}
& y^{(j)}(t, \varepsilon)=\frac{y_{30}^{(j)}(t)}{y_{30}(1)}\left(\frac{\gamma}{\bar{\delta}}-\frac{1}{\bar{\delta}} \int_{0}^{1} \frac{y_{30}(1)\left(\bar{F}(p)-\alpha \bar{K}_{1}(p, 0)\right)}{y_{30}(p) \mu_{1}(p) \mu_{2}(p)} d p\right)+ \\
& +\int_{0}^{t} \frac{y_{30}^{(j)}(t)}{y_{30}(p) \mu_{1}(p) \mu_{2}(p) y_{30}(1)}\left[\bar{F}(p)-\alpha \bar{K}_{1}(p, 0)-\frac{2 \varepsilon \beta \bar{K}_{1}(p, 0)}{\mu_{2}(0)-\mu_{1}(0)}+\right. \\
& \left.+\left(\frac{\gamma}{\bar{\delta}}-\frac{1}{\bar{\delta}} \int_{0}^{1} \frac{y_{30}(1)\left(\bar{F}(p)-\alpha \bar{K}_{1}(p, 0)\right)}{y_{30}(p) \mu_{1}(p) \mu_{2}(p)} d p\right) \frac{\bar{K}_{1}(p, 0)+\int_{0}^{1} \sum_{j=0}^{1} \bar{K}_{j}(p, x) y_{30}^{(j)}(x) d x}{y_{30}(1)}\right] d p+ \\
& +\left[\frac{\alpha y_{10}(t) \mu_{2}(0) \mu_{1}^{j}(t)}{\mu_{2}(0)-\mu_{1}(0)}+\frac{\varepsilon \beta y_{10}(t) \mu_{1}^{j}(t)}{\mu_{2}(0)-\mu_{1}(0)}-\right. \\
& \left.-\left(\frac{\gamma}{\bar{\delta}}-\frac{1}{\bar{\delta}} \int_{0}^{1} \frac{y_{30}(1)\left(\bar{F}(p)-\alpha \bar{K}_{1}(p, 0)\right)}{y_{30}(p) \mu_{1}(p) \mu_{2}(p)} d p\right) \frac{y_{10}(t) \mu_{2}(0) \mu_{1}^{j}(t)}{y_{30}(1)\left(\mu_{2}(0)-\mu_{1}(0)\right)}\right] \frac{e^{\frac{1}{\varepsilon} \int^{t} \mu_{1}(x) d x}}{\varepsilon^{j}}+ \\
& +\left[-\frac{\alpha y_{20}(t) \mu_{1}(0) \mu_{2}^{j}(t)}{\mu_{2}(0)-\mu_{1}(0)}+\frac{\varepsilon \beta y_{20}(t) \mu_{2}^{j}(t)}{\mu_{2}(0)-\mu_{1}(0)}+\right. \\
& \left.+\left(\frac{\gamma}{\bar{\delta}}-\frac{1}{\bar{\delta}} \int_{0}^{1} \frac{y_{30}(1)\left(\bar{F}(p)-\alpha \bar{K}_{1}(p, 0)\right)}{y_{30}(p) \mu_{1}(p) \mu_{2}(p)} d p\right) \frac{y_{20}(t) \mu_{1}(0) \mu_{2}^{j}(t)}{y_{30}(1)\left(\mu_{2}(0)-\mu_{1}(0)\right)}\right] \frac{e^{\frac{1}{\varepsilon}} \int_{0}^{t} \mu_{2}(x) d x}{\varepsilon^{j}}+ \\
& +\mathrm{O}\left(\varepsilon+\frac{1}{\varepsilon^{j-1}} e^{\frac{1}{\varepsilon} \int_{p}^{t} \mu_{1}(x) d x}+\frac{1}{\varepsilon^{j-1}} e^{\frac{1}{\varepsilon} \int_{p}^{t} \mu_{2}(x) d x}\right), j=\overline{0,2},
\end{aligned}
$$

where $\bar{\delta}$ has the form from condition 4. Consequently, estimating formula (45), in view of formula (30) and condition 2, we obtain the estimates (42). Thus, Theorem 4 is proved.

Definition 1. (See Reference [27]). Problem (1), (2) possesses an m-th order initial jump in the neighborhood of the point $t=0$, if its solution has the following growth character:

$$
y^{(j)}(0, \varepsilon)=\mathrm{O}(1), j=\overline{0, m}, y^{(m+j)}(0, \varepsilon)=\mathrm{O}\left(\frac{1}{\varepsilon^{j}}\right), j=\overline{1,2-m} .
$$


From Theorem 4, we directly obtain the following growth character of the solution:

$$
y(0, \varepsilon)=\mathrm{O}(1), y^{\prime}(0, \varepsilon)=\mathrm{O}(1), y^{\prime \prime}(0, \varepsilon)=\mathrm{O}\left(\frac{1}{\varepsilon^{2}}\right), \varepsilon \rightarrow 0 .
$$

Therefore, the solution of the singularly perturbed boundary value problem (1), (2) at the point $t=0$ possesses the phenomenon of an initial jump of the second degree zero-th order, according to Definition 1.

\subsection{Asymptotic Estimates of the Difference between the Solutions of the Perturbed and Unperturbed Problems}

In order to establish the asymptotic convergence of the solution, we consider whether the difference in the solutions of the perturbed and unperturbed problems tends to zero when a small parameter tends to zero.

Consider the modified unperturbed boundary value problem [27]:

$$
\begin{gathered}
L_{\varepsilon} \bar{y} \equiv B(t) \overline{y^{\prime}}+C(t) \bar{y}=F(t)+\int_{0}^{1}\left[K_{0}(t, x) \bar{y}(x)+K_{1}(t, x) \overline{y^{\prime}}(x)\right] d x+\Delta(t), \\
h_{1} \bar{y}(t) \equiv \bar{y}(0)=\alpha+\Delta_{0}, h_{3} \bar{y}(t) \equiv \bar{y}(1)=\gamma,
\end{gathered}
$$

where $\Delta(t), \Delta_{0}$ are the initial jumps of the integral term and the solution to be determined.

We introduce the function:

$$
z(t, \varepsilon)=y(t, \varepsilon)-\bar{y}(t)
$$

where $y(t, \varepsilon)$ is the solution to the problem (1), (2) and $\bar{y}(t)$ is the solution to the problem (48), (49).

Hence, substituting $y(t, \varepsilon)=z(t, \varepsilon)+\bar{y}(t)$ from equality (50) into problem (1), (2) and considering (48), (49), we obtain the equation for $z(t, \varepsilon)$

$$
\begin{aligned}
L_{\varepsilon} z & \equiv \varepsilon^{2} z^{\prime \prime \prime}+\varepsilon A(t) z^{\prime \prime}+B(t) z^{\prime}+C(t) z= \\
& =F_{\varepsilon}(t)+\int_{0}^{1}\left[K_{0}(t, x) z(x, \varepsilon)+K_{1}(t, x) z^{\prime}(x, \varepsilon)\right] d x
\end{aligned}
$$

with the boundary conditions:

$$
h_{1} z(t, \varepsilon) \equiv z(0, \varepsilon)=-\Delta_{0}, h_{2} z(t, \varepsilon) \equiv z^{\prime}(0, \varepsilon)=\beta-\bar{y}^{\prime}(0), h_{3} z(t, \varepsilon) \equiv z(1, \varepsilon)=0,
$$

where:

$$
F_{\varepsilon}(t)=-\varepsilon^{2} \bar{y}^{\prime \prime \prime}(t)-\varepsilon A(t) \bar{y}^{\prime \prime}(t)-\Delta(t) .
$$

Problem (51), (52) has the same form as problem (1), (2). Hence, applying Theorem 4 to the problem (51), (52) and considering the estimate for equality (53) and the equality (50), we obtain the estimates for $z^{(j)}(t, \varepsilon), j=\overline{0,2}$ :

$$
\begin{aligned}
\left|z^{(j)}(t, \varepsilon)\right| & \leq C\left[\varepsilon\left|\beta-\bar{y}^{\prime}(0)\right|+\max _{0 \leq t \leq 1}\left|\Delta(t)-\Delta_{0} K_{1}(t, 0)\right|\right]+ \\
& +\frac{C}{\varepsilon^{\prime}} e^{-\delta \frac{t}{\varepsilon}}\left[\left(\left|\Delta_{0}\right|+\max _{0 \leq t \leq 1}\left|\Delta(t)-\Delta_{0} K_{1}(t, 0)\right|\right)\left|\mu_{2}(0) \mu_{1}^{j}(t)-\mu_{1}(0) \mu_{2}^{j}(t)\right|+\varepsilon\left|\beta-\bar{y}^{\prime}(0)\right|\right], j=\overline{0,2 .}
\end{aligned}
$$

It follows from estimates (54) under the condition

$$
\Delta(t)=\Delta_{0} K_{1}(t, 0)
$$

that $z^{(j)}(t, \varepsilon) \rightarrow 0, j=\overline{0,2}, 0<t \leq 1$.

Hence, the following theorem is true. 
Theorem 5. If conditions 1-4 are satisfied, then, for sufficiently small $\varepsilon>0$, the following asymptotic estimates are true for the difference $y(t, \varepsilon)-\bar{y}(t)$ between the solution of problem (1), (2) and the solution of problem (48), (49) on the segment $[0,1]$ :

$$
\left|y^{(j)}(t, \varepsilon)-\bar{y}^{(j)}(t)\right| \leq C \varepsilon+\frac{C}{\varepsilon^{j}} e^{-\delta \frac{t}{\varepsilon}}\left|\mu_{2}(0) \mu_{1}^{j}(t)-\mu_{1}(0) \mu_{2}^{j}(t)\right|, 0<t \leq 1, j=\overline{0,2},
$$

where $C>0$ are constants independent of $\varepsilon$.

It follows from Theorem 5 that the solution of the singularly perturbed problem (1), (2) tends to the solution of the modified unperturbed problem (48), (49). From estimates (56), we obtain the following limit equalities:

$$
\lim _{\varepsilon \rightarrow 0} y^{(j)}(t, \varepsilon)=\bar{y}^{(j)}(t), 0<t \leq 1, j=\overline{0,2}
$$

We notice that the limit transitions (57) are not uniform in the neighborhood of the point $t=0$. They are uniform on the segment $0<t_{0} \leq t \leq 1$, where $t_{0}$ is a sufficiently small but fixed number as $\varepsilon \rightarrow 0$.

\section{Conclusions}

In this paper, we considered a two-point boundary value problem for a linear integro-differential equation of the third order with a small parameter at two higher derivatives, when the roots of the additional characteristic equation are negative. Asymptotic representations of the fundamental system of solutions, the Cauchy function, and the boundary functions of a singularly perturbed homogeneous differential equation were obtained. Theorems on the existence and uniqueness of a solution, on asymptotic estimates of the solution and its derivatives, and on estimates of the difference between the solutions of a singularly perturbed and unperturbed problem were proved. The asymptotic convergence and the limit equality of the solution of this problem to the solution of the modified unperturbed boundary value problem were established. Further study of this problem is aimed at constructing an asymptotic expansion of the given unperturbed problem as the small parameter tends to zero. All scientific results obtained in solving singularly perturbed problems have theoretical and practical significance and can be applied to a qualitative study or practical solution of certain problems of physics, mechanics, and other fields of science.

Author Contributions: Conceptualization, A.Z.; funding acquisition, Y.I.C.; supervision, Y.I.C.; writing一original draft preparation, A.Z. All authors have read and agreed to the published version of the manuscript.

Funding: This research was supported by the MSIT, Korea, under the ITRC support program (IITP-2019-2017-0-01630) supervised by the IITP and in part by the NRF-2018R1D1A1A09084151.

Acknowledgments: The authors are grateful to the referees for useful reviews and comments on improving the paper.

Conflicts of Interest: The authors declare no conflict of interest.

\section{References}

1. Vasilyeva, A.B.; Butuzov, V.F. Asymptotic Methods in the Theory of Singular Perturbations; Visshaya shkola: Moscow, Russia, 1990.

2. Friedrichs, K.O. Asymptotic phenomena in mathematical physics. Mathematics 1957, 1, 79-94. [CrossRef]

3. Kato, T. Perturbation Theory for Linear Operators; MIR: Moscow, Russia, 1972.

4. Tikhonov, A.N. On systems of differential equations containing parameters. Matem. Sbor. 1950, 27, 147-156.

5. Tikhonov, A.N. Systems of differential equations containing parameters at derivatives. Matem. Sbor. 1952, 31, 575-586.

6. Lomov, S.A. Introduction to the General Theory of Singular Perturbations; Nauka: Moscow, Russia, 1981.

7. Lomov, S.A. Perturbation method for singular problems. Dokl. Akad. Nauk Arch. 1972, 36, 635-651. [CrossRef] 
8. Lomov, S.A. On a method of regularization of singular perturbations. Dokl. Akad. Nauk Arch. 1967, 177, 1273-1275.

9. Vishik, M.I.; Lyusternik, L.A. Regular degeneration and a boundary layer for linear differential equations with small parameter. Uspekhi Mat. Nauk 1957, 12, 3-122.

10. Vasilyeva, A.B. Asymptotic methods in the theory of ordinary differential equations with small parameters at the higher derivatives. USSR Comput. Math. Math. Phys. 1963, 3, 611-642. [CrossRef]

11. Imanaliev, M.I. Asymptotic Methods in the Theory of Singularly Perturbed Integro-Differential Systems; Ilim: Frunze, Kyrgyzstan, 1972.

12. Imanaliev, M.I. Oscillations and Stability of Solutions of Singularly Perturbed Integro-Differential Systems; Ilim: Frunze, Kyrgyzstan, 1974.

13. Butuzov, V.F. On the question of the asymptotic of solutions of integro-differential equations with a small parameter at the derivative. Differ. Equ. 1966, 2, 391-406.

14. Butuzov, V.F. Corner boundary layer in singularly perturbed problems with partial derivatives. Differ. Equ. 1979, 15, 1848-1862.

15. Vazov, V. Asymptotic Expansions of Solutions of Ordinary Differential Equations; MIR: Moscow, Russia, 1968.

16. Nayfe, A.K. Perturbation Methods; MIR: Moscow, Russia, 1976.

17. Cole, D.J. Perturbation Methods in Applied Mathematics; MIR: Moscow, Russia, 1972.

18. Bogolyubov, N.N.; Mitropolsky, Y.A. Asymptotic Methods in the Theory of Nonlinear Oscillations: Mathematics and Nonlinear Mechanics; Nauka: Moscow, Russia, 2005.

19. Mishchenko, E.F.; Rozov, N.K. Differential Equations with a Small Parameter and Relaxation Oscillations; Nauka: Moscow, Russia, 1975.

20. Vishik, M.I.; Lyusternik, L.A. On the initial jump for nonlinear differential equations containing a small parameter. Dokl. Akad. Nauk Arch. 1960, 132, 1242-1245.

21. Kasymov, K.A. On the asymptotic of the solution of the Cauchy problem with large initial conditions for nonlinear ordinary differential equations containing a small parameter. Uspekhi Mat. Nauk 1962, 17, 187-188.

22. Kasymov, K.A. Asymptotics of the solution of the problem with an initial jump for nonlinear systems of differential equations with a small parameter with the highest derivative. Dokl. Akad. Nauk Arch. 1969, 189, 941-944.

23. Kasymov, K.A. Singularly Perturbed Boundary Value Problems with Initial Jumps; Sanat: Almaty, Kazakhstan, 1997.

24. Nurgabyl, D. Asymptotic estimates for the solution of a restoration roblem with initial jump. J. Appl. Math. 2014, 2014, 1-11. [CrossRef]

25. Nurgabyl, D.; Boribekova, F.; Nurgabylova, G. Boundary value problems with boundary jumps for singularly perturbed differential equations of conditionally stable type in the critical case. Glob. J. Pure Appl. Math. 2016, 12, 3425-3432.

26. Kasymov, K.A.; Dauylbaev, M.K. On the estimation of solutions of the Cauchy problem with an initial jump of any order for linear singularly perturbed integro-differential equations. Differ. Equ. 1999, 35, 822-830.

27. Kasymov, K.A.; Dauylbaev, M.K. Singularly perturbed linear integro-differential equations with initial jumps of any order. Izv. vuzov Math. 2003, 7, 70-74.

28. Dauylbaev, M.K. The asymptotic behavior of solutions to singularly perturbed nonlinear integro-differential equations. Siber. Math. J. 2000, 41, 49-60. [CrossRef]

29. Dauylbaev, M.K.; Nurgabyl, D.; Atakhan, N. Asymptotic expansion of solutions of boundary value problems with initial jumps for singularly perturbed integro-differential equations. Bull. KazNU 2014, 83, 43-51.

30. Dauylbaev, M.K.; Atakhan, N. The initial jumps of solutions and integral terms in singular boundary value problem of linear higher order integro-differential equations. Misk. Math. Notes 2015, 16, 747-761. [CrossRef]

31. Dauylbaev, M.K.; Dzhumabaev, D.S.; Atakhan, N. Asymptotical representation of singularly perturbed boundary value problems for integro-differential equations. J. NAS RK 2017, 2, 18-26.

32. Shabat, A.B. Boundary value problems with a small parameter for ordinary linear differential equations. Uspekhi Mat. Nauk 1962, 17, 235-241.

(C) 2020 by the authors. Licensee MDPI, Basel, Switzerland. This article is an open access article distributed under the terms and conditions of the Creative Commons Attribution (CC BY) license (http://creativecommons.org/licenses/by/4.0/). 